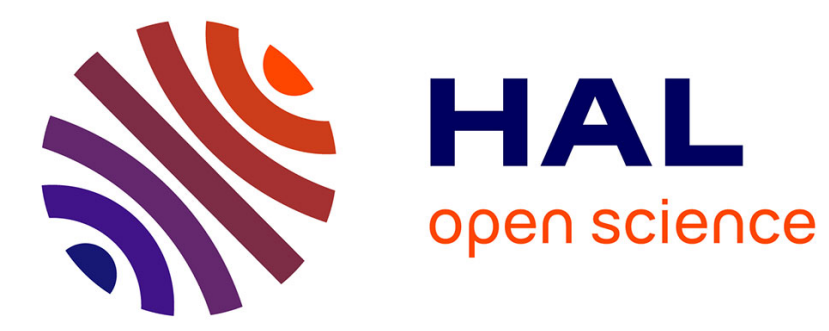

\title{
Pluviométrie à l'île de la Réunion: des travaux de J. Defos du Rau (1960) à nos jours
}

René Robert

\section{To cite this version:}

René Robert. Pluviométrie à l'île de la Réunion: des travaux de J. Defos du Rau (1960) à nos jours. L'Information géographique, 2001, 65 (1), pp.53 - 59. 10.3406/ingeo.2001.2734 . hal-03446644

\section{HAL Id: hal-03446644 \\ https://hal.univ-reunion.fr/hal-03446644}

Submitted on 24 Nov 2021

HAL is a multi-disciplinary open access archive for the deposit and dissemination of scientific research documents, whether they are published or not. The documents may come from teaching and research institutions in France or abroad, or from public or private research centers.
L'archive ouverte pluridisciplinaire HAL, est destinée au dépôt et à la diffusion de documents scientifiques de niveau recherche, publiés ou non, émanant des établissements d'enseignement et de recherche français ou étrangers, des laboratoires publics ou privés.

\section{(1) (1) $\$$}

Distributed under a Creative Commons Attribution - NonCommercial - NoDerivatives 44.0 


\section{Pluviométrie à l'île de la Réunion: des travaux de J. Defos du Rau (1960) à nos jours}

\section{René Robert}

\section{Citer ce document / Cite this document :}

Robert René. Pluviométrie à l'île de la Réunion: des travaux de J. Defos du Rau (1960) à nos jours. In: L'information géographique, volume $65, \mathrm{n}^{\circ} 1,2001$. pp. 53-59;

doi : https://doi.org/10.3406/ingeo.2001.2734

https://www.persee.fr/doc/ingeo_0020-0093_2001_num_65_1_2734

Fichier pdf généré le 09/05/2018 


\begin{abstract}
In the very first geographic study of Reunion island, Defos du Rau's study pointed out two rainbelts, defined by the $2000 \mathrm{~mm}$ per annum mark. The windward region includes the eastern side, the middle cirques, and a some of the western slopes. Takamaka owns the highest recorded rainfall while the la Fournaise's eastern slopes rains were not studied thoroughly. At present, the map takes into account the yearly median. The windward region has been restricted to the eastern part, plus the cirque of Salazie. As for the rainfall peaks they are actually recorded along the eastern slopes of the la Fournaise volcano. On the other land, the areas registering more than $2000 \mathrm{~mm} /$ year are quite limited. Similarly, the high mountains (over $2500 \mathrm{~m}$ higher) receive less than $1000 \mathrm{~mm}$ per year.
\end{abstract}

\title{
Résumé
}

La première étude de géographie de l'île de la Réunion (Defos du Rau, 1960 ) propose une carte des pluies avec deux régions, au vent et sous le vent, de part et d'autre de l'isohyète moyenne annuelle 2 $000 \mathrm{~mm}$. La région au vent correspond à la façade orientale de nie avec un pôle de la pluie situé à Takamaka ; la région sous le vent, à la façade occidentale. Les pluies du massif volcanique de la Fournaise n'étaient pas mesurées. Aujourd'hui, la carte des médianes annuelles de pluie fait apparaître une région au vent réduite, avec un maximum sur les pentes moyennes orientales de la Fournaise. Ailleurs les pluies supérieures à $2000 \mathrm{~mm} / \mathrm{an}$ sont rares ; les hautes terres, au- dessus de $2500 \mathrm{~m}$, reçoivent moins de $1000 \mathrm{~mm} / \mathrm{an}$. 


\title{
Pluviométrie à l'île de la Réunion: des travaux de J. Defos du Rau (1960) à nos jours
}

\author{
René Robert \\ Professeur de géographie, Université de la Réunion \\ Responsable du laboratoire de géographie de l'environnement naturel
}

\begin{abstract}
La première étude de géographie de l'île de la Réunion (Defos du Rau, 1960) propose une carte des pluies avec deux régions, au vent et sous le vent, de part et d'autre de l'isohyète moyenne annuelle $2000 \mathrm{~mm}$. La région au vent correspond à la façade orientale de l'île avec un pôle de la pluie situé à Takamaka ; la région sous le vent, à la façade occidentale. Les pluies du massif volcanique de la Fournaise n'étaient pas mesurées. Aujourd'hui, la carte des médianes annuelles de pluie fait apparaître une région au vent réduite, avec un maximum sur les pentes moyennes orientales de la Fournaise. Ailleurs les pluies supérieures à 2000 $\mathrm{mm} / \mathrm{an}$ sont rares; les hautes terres, audessus de $2500 \mathrm{~m}$, reçoivent moins de $1000 \mathrm{~mm} / \mathrm{an}$.
\end{abstract}

Mots-clés: océan Indien, la Réunion, massif du Piton-des-Neiges, massif de la Fournaise, région au vent, région sous le vent, pluviométrie.

\section{INTRODUCTION}

Le relief de l'île de la Réunion est fait de deux massifs volcaniques, l'un au nord-ouest, le Piton-des-Neiges, et l'autre toujours en activité dans le sudest, la Fournaise. Ces constructions accolées offrent une architecture de
In the very first geographic study of Reunion island, Defos du Rau's study pointed out two rainbelts, defined by the $2000 \mathrm{~mm}$ per annum mark. The windward region includes the eastern side, the middle cirques, and a some of the western slopes. Takamaka owns the highest recorded rainfall while the la Fournaise's eastern slopes rains were not studied thoroughly. At present, the map takes into account the yearly median. The windward region has been restricted to the eastern part, plus the cirque of Salazie. As for the rainfall peaks they are actually recorded along the eastern slopes of the la Fournaise volcano. On the other land, the areas registering more than $2000 \mathrm{~mm} /$ year are quite limited. Similarly, the high mountains (over $2500 \mathrm{~m}$ higher) receive less than $1000 \mathrm{~mm}$ per year.

Key-words: Indian ocean, Reunion island, Piton des Neiges volcano, la Fournaise volcano, windward region, eastern slopes, rainfall.

deux cônes surbaissés, le premier culminant à $3070 \mathrm{~m}$ et l'autre à $2632 \mathrm{~m}$. Les flux atmosphériques viennent buter contre cette «montagne dans l'océan». Tout au long de l'année c'est le flux des alizés généré par l'anticyclone de l'océan indien toujours centré au sud de 
la Réunion: il est le plus fréquemment orienté ESE. De temps en temps la circulation des alizés est perturbée. En saison chaude surtout (de novembre à avril), c'est l'arrivée d'une perturbation tropicale, issue de la ZCIT et qui dans sa trajectoire se rapproche plus ou moins de l'île. Selon sa position elle dirige des vents de différents secteurs et elle est surtout responsable d'importantes pluies. Irrégulièrement dans l'année, venant du lointain sud, une perturbation d'origine polaire, un front froid, traverse l'île en y déversant des pluies. L'alimentation des régions de l'île est assez hétérogène, en raison de cette combinaison de pluviogenèses, et du rôle joué par les massifs montagneux.

L'installation des stations pluviométriques date surtout des années 19501960. Au début, elle a été faite en fonction de la présence quotidienne d'un responsable. Les hauts de l'île et la façade est du massif de la Fournaise, régions inhabitées et difficiles d'accès, n'ont pas été équipés dans un premier temps. Lors de ses recherches menées au cours des années 1950 Jean Defos du Rau ne dispose que d'un réseau incomplet et d'une série statistique assez courte. La carte publiée dans la thèse de 1960 est adaptée aux connaissances et moyens d'une époque pionnière.

À partir de 1967 l'île est dotée de moyens lui permettant de recevoir des images satellitaires. Les stations au sol bénéficient d'améliorations techniques: certaines sont aujourd'hui totalement automatiques. La couverture de l'espace réunionnais s'est nettement améliorée, notamment dans les hauts et dans la Fournaise. Les résultats sont assez surprenants, tant dans l'importance des valeurs mesurées que dans la distribution géographique des pluies.

\section{LA CARTE DES PLUIES DE 1960}

La carte des pluies a été réalisée à partir d'une quarantaine de stations dont les plus significatives sont portées sur la carte (fig. 1): les régions de canne à sucre du nord-est et du sud-ouest de l'île, les principales villes du littoral, les pentes moyennes de l'ouest, l'axe transversal de Saint-Benoit à Saint-Pierre par le plateau central de la Plaine des Cafres, sont équipés de pluviomètres. Dans les hauts, cela n'est pas le cas. Il y a peu d'installations dans les cirques du Piton-des-Neiges, et de très rares postes sur les pentes du même massif au-dessus de 1000 mètres. L'absence de poste est notoire dans le massif de la Fournaise au-dessus de 1000 mètres.

La limite entre les régions au vent et sous le vent à partir des moyennes annuelles a été définie par l'jsohyète $2000 \mathrm{~mm}$. La région au vent se situe à l'est et au centre de l'île: façade orientale et sommets du massif de la Fournaise; façade orientale, ensemble des sommets, cirques centraux, et partie sommitale de la façade occidentale du massif du Piton-des-Neiges. Elle est alimentée principalement par les pluies d'alizés, et irrégulièrement par les pluies de cyclones tropicaux.

La région sous le vent s'étend sur les littoraux et pentes basses du nord (région de Saint-Denis) au sud de l'île (région de Saint-Joseph), en passant par l'ouest (région de Saint-Gilles).

Les pôles de la pluie sont Takamaka et Saint-Gilles. Le site de Takamaka est particulier: dans le sud-est du massif du Piton-des-Neiges, au fond d'une vallée encaissée, celle de la rivière des Marsouins, à une altitude de 649 mètres. Cette région était connue comme très pluvieuse et les débits de la rivière avaient donné l'idée dès les années 1930 de l'installation d'une cen- 
Fig. 1 : Carte des pluies d'après les travaux de Defos du Rau

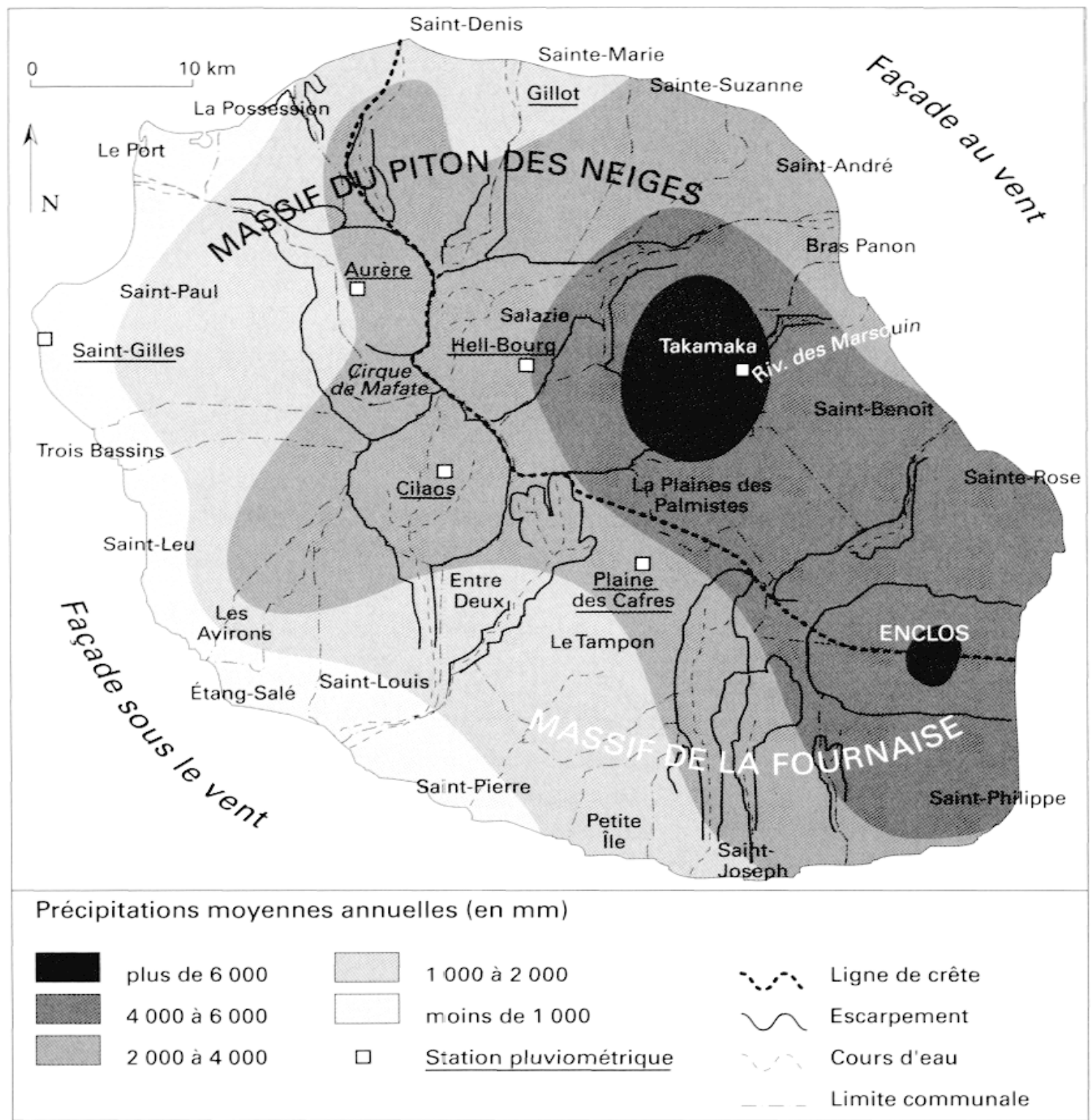

trale hydroélectrique. En 1952, à la suite de pluies diluviennes, particulièrement en mars (passage d'une dépression tropicale), la somme des pluies avait été supérieure à 14 mètres. La moyenne annuelle de la période est de $8000 \mathrm{~mm}$. Saint-Gilles est la plus connue des stations balnéaires, dans l'ouest de l'île. Elle est réputée pour son ensoleillement. La moyenne annuelle de la période est de l'ordre de $550 \mathrm{~mm}$. À une quarantaine de $\mathrm{km}$ de distance à vol d'oiseau, Takamaka reçoit environ 14,5 fois plus de pluie que Saint-Gilles. Faute de stations, les pluies sur la façade orienta- le de la Fournaise ne sont pas connues. Leur estimation est rendue difficile par l'absence presque totale d'écoulement dans la région en raison d'une perméabilité très importante (coulées largement diaclasées, coulées de structure scoriacée).

\section{LA CARTE ACTUELLE DES PLUIES}

En quarante ans beaucoup de stations ont été installées; certaines ont été fermées. Beaucoup de pluviographes ont été installés par Météo-France, grâce à 
l'ouverture de nouvelles voies d'accès vers les hautes terres de l'île. C'est le cas de la station des Hauts de SainteRose, ouverte en 1973, au moment de la construction de la centrale hydroélectrique de Sainte-Rose: elle a montré pour la première fois l'importance méconnue des pluies sur la façade orientale de la Fournaise. C'est le cas pour la station de Bellecombe, à $2250 \mathrm{~m}$, au terminus de la route forestière du Volcan (c'est la station la plus haute de l'île à l'époque!). C'est aussi le cas pour celle du Maïdo, dans les hauts du nord-ouest, à $2200 \mathrm{~m}$, au-dessus du cirque de Mafate. C'est l'ensemble des stations automatiques du réseau de MétéoFrance. Pour les travaux de recherches doctorales (Barcelo, 1996), le laboratoire universitaire des Sciences de la Terre a équipé ponctuellement la façade orientale de la Fournaise.

Avec une somme de données nouvelles, la carte des pluies ne pouvait qu'être précisée. Mais surtout le choix des médianes annuelles (à la place des moyennes) apporte une différence dans la conception de la carte. Ce choix est lié à l'importance relative des pluies irrégulières de perturbations tropicales (PT). À partir de la carte de l'analyse des pourcentages de pluies de PT par rapport au total général des pluies (fig. 2), l'île se divise en trois régions.

La première a dans son total moyen annuel moins de $33 \%$ d'alimentation par les pluies de PT. Il s'agit des régions littorales et premières pentes orientales

Fig. 2: Carte de distribution régionale des pourcentages d'apport des pluies de perturbations tropicales

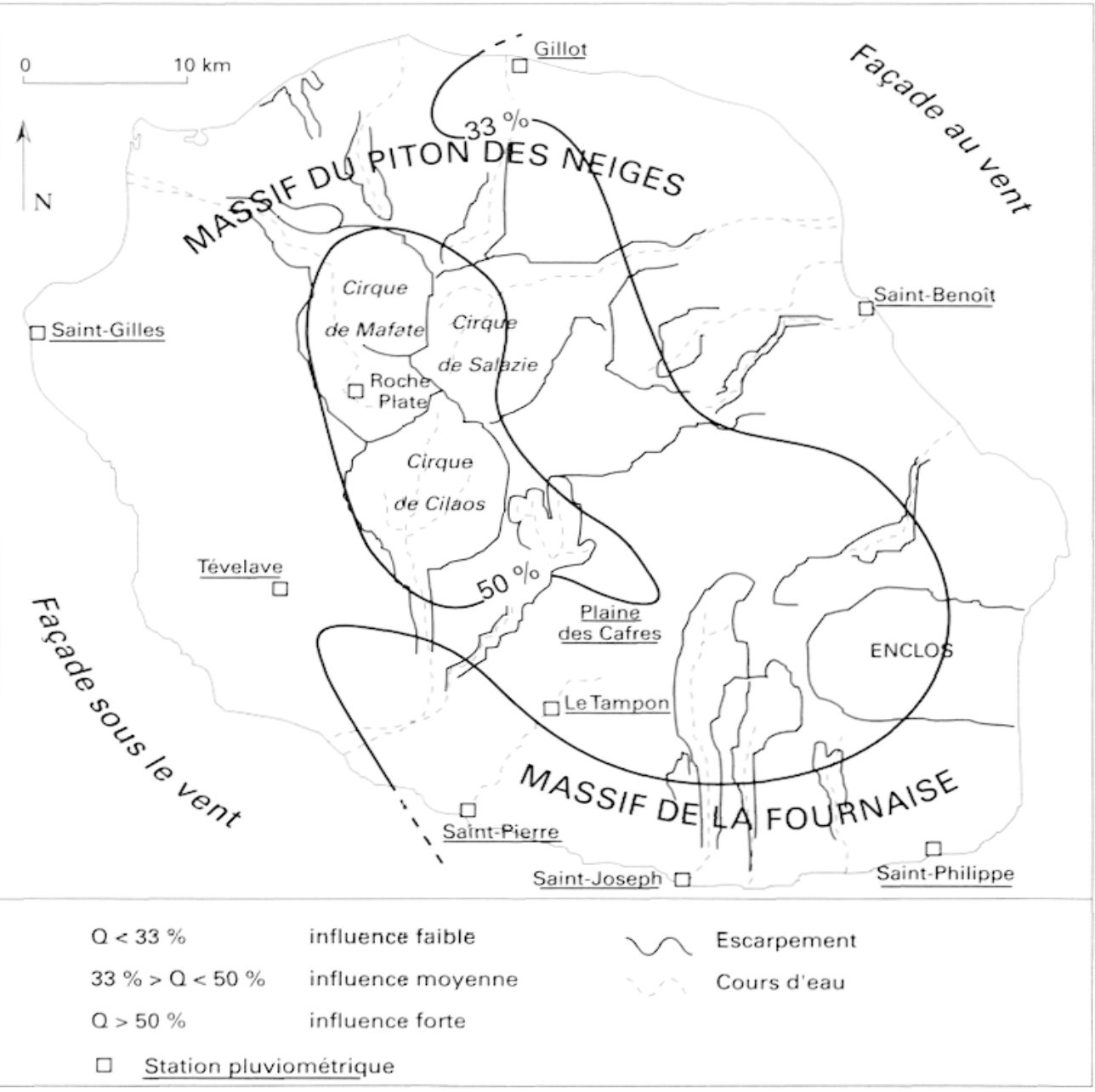


de Gillot au nord jusqu'à Saint-Joseph dans le sud. C'est la région très fortement et très régulièrement arrosée par les pluies d'alizés. C'est la région la plus faiblement influencée par les pluies de PT, surtout la région littorale est de la Fournaise de Saint-Benoit à SaintPhilippe (qui a également les plus faibles pourcentages de pluies de PT, moins de $25 \%$ ). C'est aussi un ensemble de régions littorales et premières pentes du sud de Saint-Joseph à la région du Tévelave: elle est nettement moins influencée par les pluies d'alizés que la région précédente mais elle reçoit beaucoup plus de pluies par passage de front froid (bonne exposition au sud).

La deuxième région de l'île est a contrario fortement dépendante des pluies de PT pour son alimentation moyenne annuelle ( $Q>50 \%)$. Il s'agit prioritairement des cirques du Pitondes-Neiges (Mafate, Cilaos et la plus grande partie de Salazie). Ces grands encaissements sont à l'origine d'ascendances forcées quand les vents cycloniques s'y engouffrent, provoquant une forte intensité des pluies. Il est à noter que les deux cirques occidentaux, Cilaos et surtout Mafate, moins bien arrosés par les pluies d'alizés, ont une alimentation optimale par les pluies de PT (68\% à la station de Roche Plate). Le cas du haut plateau central de la Plaine des Cafres est particulier: l'importance relative des pluies de PT est à mettre en relation avec la diminution rapide des pluies d'alizés en relation avec l'organisation progressive d'une subsidence de l'air vers les pentes sud du Tampon vers Saint-Pierre.

La troisième région est une région intermédiaire.

Les régions climatiques au vent et sous le vent sont définies par la médiane annuelle $2000 \mathrm{~mm}$. Elles sont en superficie assez nettement différentes de celles définies par la carte de Defos du
Rau. L'aire de la région au vent est plus petite (par rapport à celle de 1960): elle ne concerne plus les cirques occidentaux du Piton-des-Neiges (Mafate et Cilaos) et une partie du cirque de Salazie. Les hautes pentes du Piton-des-Neiges en sont exclues, et aussi l'ensemble des hautes pentes de la façade occidentale. La région sous le vent a des dimensions plus importantes que dans le cas précédent. Cela est dû à l'amélioration du parc de stations météorologiques, au choix de la médiane qui diminue le poids des pluies irrégulières de PT, à la prise en compte de la structure verticale des couches d'alizés (couche humide au contact de l'océan, couche d'inversion, couche supérieure plus sèche).

Le pôle de la pluie n'est plus le même et l'importance des valeurs annuelles de la Fournaise orientale peut surprendre. Les travaux de Barcelo ont révélé des sommes considérables. La présence de la station des Hauts de Sainte-Rose (Robert, 1986) avait déjà montré des moyennes annuelles supérieures à $10000 \mathrm{~mm}$. L'installation des stations nouvelles a mis en évidence des sommes annuelles supérieures à $12000 \mathrm{~mm}$, et sans doute plus. Pour ce qui concerne le pôle, les maximums de pluie connus se situent dans une tranche altitudinale comprise entre 1700 et $1900 \mathrm{~m}$ dans la caldeira de l'Enclos. C'est environ $200 \mathrm{~m}$ au-dessus de l'altitude de l'optimum pluviométrique ailleurs dans l'île. La couche d'inversion des alizés semble ici se situer à une altitude supérieure. peut-être par effets d'accumulation et de compression des masses d'air, venus du large, au contact des fortes pentes du Volcan. En 1995 la station à 1900 M. dans la caldeira de l'Enclos a enregistré un record pour l'île à plus de $20000 \mathrm{~mm}$. Depuis cette date, quelques statistiques récoltées par l'Observatoire Volcanologique de la Fournaise, ont confirmé ces valeurs extrêmes. Le pôle des plus faibles pluies reste sur le littoral ouest, de Saint-Leu à Saint-Gilles. 
Fig. 3 : Carte actuelle des pluies

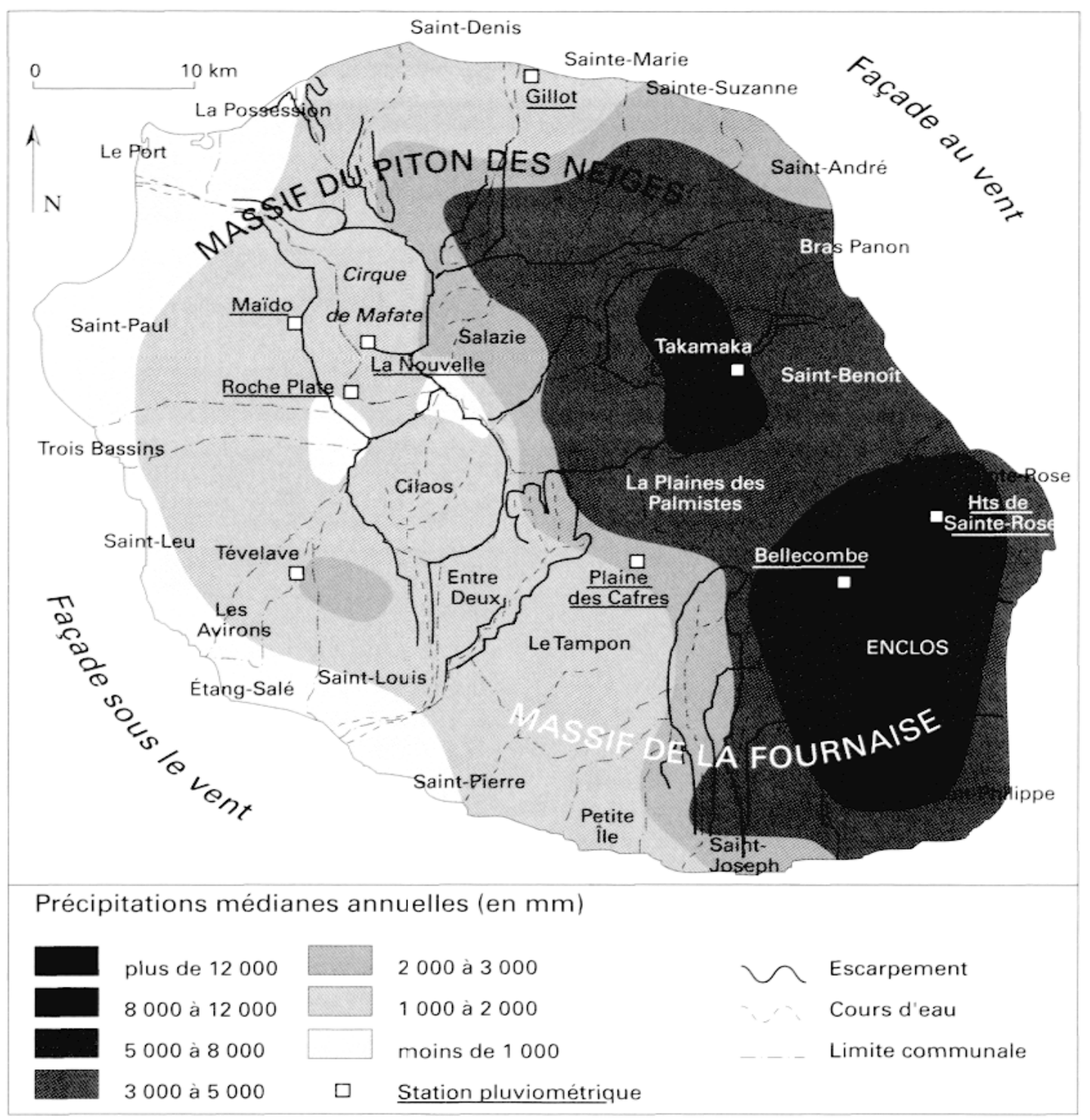

Cette carte propose une autre nouveauté : il s'agit d'une extrapolation pour les régions au-dessus de 2500 mètres dans le massif du Piton-des-Neiges uniquement. Compte tenu de la structure de la couche d'alizés (Cabaussel, 1988), l'alimentation des hautes terres centrales par les pluies de régime d'alizés est faible. Les mesures effectuées depuis peu dans les hauts de l'ouest le confirment (moins de $600 \mathrm{~mm}$ en 1999, pour une station située à $1800 \mathrm{~m}$ ). La forte irrégularité de l'alimentation par les pluies de PT laisse présager des valeurs de médianes annuelles assez faibles. Il est ici proposé qu'au-dessus de $2500 \mathrm{~m}$ ces valeurs médianes soient inférieures à
$1000 \mathrm{~mm} / \mathrm{an}$. Cela reste à vérifier. Du côté de la Fournaise, les conditions semblent différentes, comme le prouvent les statistiques de Bellecombe, riches en pluies d'alizés, et la position haute de la couche d'inversion par rapport à celle qui a été observée par ailleurs sur les pentes du massif du Piton-des-Neiges.

\section{CONCLUSION}

D'importantes précisions ont été apportées depuis 1960. Deux aires géographiques restent toutefois à considérer d'une manière précise: la façade orientale de la Fournaise, les hautes terres de l'île au-dessus de 2000 m. 
Les difficultés d'installation et de maintenance des stations dans la région de l'Enclos (caldeira de la Fournaise où se font $99 \%$ des coulées depuis la période historique et pentes proches couvertes de forêt luxuriante) restent importantes. Les travaux de Barcelo n'ont duré que trois années (1993-1995) et demandent à être confirmés. Des stations pourraient être réinstallées en relation avec l'Observatoire Volcanologique. Nul doute que les résultats acquis risquent d'être corrigés à la hausse dès le moment où les trois années d'étude n'ont pas connu de perturbations tropicales très riches en pluies intenses et durables.

Pour ce qui concerne les hautes terres, une action est en cours actuellement: elle concerne la présence d'une «nebelwald» dans les hauts de l'ouest de l'île. Trois stations automatiques ont été installées à 1600,1800 , et 2250 mètres. Les résultats obtenus au cours de la première année révèlent des caractéristiques climatiques particulières à ces régions, et notamment une sécheresse bien plus sévère que ce qui était prévu.

En deçà de la connaissance scientifique de la distribution des pluies dans l'île de la Réunion, les travaux de climatologie sur la façade au vent de la Fournaise et dans les hauts de l'île cherchent à répondre à une problématique, celle des disponibilités en eau pour la société réunionnaise. Les sommes remarquables de pluies annuelles sur la Fournaise ne donnent, par exemple dans la caldeira de l'Enclos, aucun écoulement de surface, en raison d'une perméabilité maximale. Ailleurs, sur la façade orientale de la Fournaise, les écoulements sont sporadiques (quelques crues dans l'année, de faible durée). Le château d'eau de la Fournaise enregistre des records du monde de pluie et ne fournit pas beaucoup d'eau utile (à moins de trouver des solutions d'ingénierie pour ramener artificiellement l'eau à la surface!). Par ailleurs dans les hauts de l'île, il est prévu la mise en place d'un parc naturel. Le frein premier à la valorisation des remarquables paysages de ce futur parc est incontestablement le manque d'eau.

\section{Bibliographie}

A. Barcelo, Analyse des mécanismes hydrologiques en domaine volcanique insulaire tropical à relief jeune: apports à la connaissance du bilan hydrique (massif du Piton de la Fournaise, île de la Réunion), Université de la Réunion, 1996, 266 p.

A. Barcelo, R. Robert, J. Coudray, A major rainfall event: the 27 february-5 march 1993 rains on the southeastern slope of Piton de la Fournaise massif (Reunion island, Indian ocean), American Meteorological Society, Notes and Correspondence, 1996, p. 33413346.

A. Barcelo, R. Robert, J. Coudray, «Les pluies sur les versants orientaux du massif de la Fournaise (île de la Réunion, océan indien)", Bulletin de l'Association des Géographes Français, Paris, 3, 1998, p. 371-380.

G. Cabaussel, Les caractères originaux de la climatologie dans les montagnes tropicales, Actes du $\mathrm{IX}^{\mathrm{e}}$ colloque de la SÉPANRIT, Bordeaux, 1988, p. 15-23.

Defos du Rau. L'île de la Réunion, étude de géographie humaine. Bordeaux, 1960, 716 p.

Météo-France, Atlas climatique de la Réunion, Saint-Denis, Éditions MétéoFrance, Direction interrégionale de la Réunion. 1997.80 p.

R. Robert. Climat et hydrologie à la Réunion: étude typologique et régionale des pluies et de l'écoulement. Sainte-Clotilde, la Réunion, Dyonisienne. 1986, 438 p.

R. Robert. «Les pluies de grande intensité à la Réunion". Bulletin de la Société Languedocienne de géographie. Montpellier, spécial Catastrophes et Risques naturels. 1990, 1-2. p. 119-132.

R. Robert. L'impact des cyclones tropicaux dans les DOM-TOM. Les Français dans leur emironnement, Paris, Nathan, 1993, p. 125-130. 